\section{Deficiencia de glucosa 6-fostato deshidrogenasa en hombres sanos y en pacientes maláricos; Turbo (Antioquia, Colombia)}

\section{Deficiency of glucose-6-phosphate dehydrogenase in healthy men and malaria patients; Turbo (Antioquia, Colombia)}

\section{Jaime Carmona-Fonseca \\ Gonzalo Álvarez \\ Alexandra Ríos \\ María Fernanda Vásquez}

Grupo Salud y Sociedad, Universidad de Antioquia - http://medicina.udea.edu. co/Investigacion/Grupos/malaria/index.htm

Financiación: Colciencias (contrato RC-253-2004 ), Dirección Seccional de Salud de Antioquia (contrato Cl-270-2004) y Universidad de Antioquia.

Agradecimientos: A los directivos, empleados y pacientes del "Hospital Francisco Valderrama" y del "Puesto de salud San José" de Turbo. A los médicos, laboratoristas y demás contratistas de los proyectos.

Correspondencia: Jaime Carmona-Fonseca. Calle 62 52-59, laboratorio 610, Medellín (Colombia)

E-mail: jaimecarmonaf@hotmail.com

\section{Resumen}

Introducción: En América Latina la deficiencia de glucosa 6 -fosfato deshidrogenasa (d-G6PD) ha sido poco estudiada y en Colombia solo conocemos tres publicaciones antiguas. Urge conocer más la prevalencia de d-G6PD, sobre todo ahora que el tratamiento de la malaria vivax plantea aumentar la dosis diaria o total de primaquina. Objetivo: Medir la prevalencia de d-G6PD en poblaciones masculina sana y de enfermos con malaria por Plasmodium vivax, en Turbo (Urabá, departamento de Antioquia, Colombia). Metodología: Encuestas de prevalencia, para evaluar la G6PD en dos poblaciones de Turbo (Antioquia): hombres sanos; hombres y mujeres con malaria vivax. Se trabajó con muestras diseñadas con criterios estadístico-epidemiológicos. La actividad enzimática se midió con el método normalizado de Beutler para valorar la G6PD en hemolizados. Resultados: Entre los hombres sanos $(\mathrm{n}=508)$, el intervalo de confianza 95\% para el promedio (IC95\%) estuvo entre 4,15 y 4,51 UI/g hemoglobina y $14,8 \%$ presentaron valores por debajo del "límite normal" de <2,29 UI/g hemoglobina (prevalencia de d-G6PD). Entre los hombres con malaria $(n=206)$ el IC95\% fue 3,81 a $4,16 \mathrm{UI} / \mathrm{g}$ hemoglobina y entre las mujeres palúdicas fue 3,86 a 4,20 UI/g hemoglobina. Los promedios masculinos (sanos $v s$. maláricos) fueron estadísticamente diferentes $(\mathrm{p}=0,028)$. Únicamente 9,5\% (13/137) de los enfermos con paludismo, todos de sexo masculino, presentaron d-G6PD. Conclusiones: la d-G6PD es relativamente alta $(14,8 \%)$ en la población masculina sana de Turbo y en los enfermos maláricos por $P$. vivax (9,5\%, todos hombres).

Palabras-clave: Glucosa deshidrogenasas. Malaria, paludismo. Plasmodium. Etnia. Sexo. Primaquina. Colombia 


\section{Abstract}

Introduction: Glucose-6-phosphate dehydrogenase (G6PD) deficiency in Latin America has not been fully studied and in Colombia only three outdated publications are known. Recent information on the prevalence of G6PD deficiency is required now, because the recommended treatment of vivax malaria requires higher daily or total doses of primaquine. Objective: To measure the prevalence of G6PD in a healthy male population and in a Plasmodium vivax infected population in Turbo (Urabá, Antioquia Department, Colombia). Method: Prevalence survey to evaluate G6PD in two populations of Turbo (Antioquia): healthy male; male and female with vivax malaria. The work was carried out on population samples selected using statistical and epidemiological criteria. Enzyme activity was measured using Beutler's normalized method to evaluate G6PD after hemolysis. Results: For the healthy male group ( $\mathrm{n}=$ 508), and with a $95 \%$ confidence interval (IC95\%), the mean values were between 4.15 and $4.51 \mathrm{IU} / \mathrm{g}$ hemoglobin; $14.8 \%$ exhibited values below "normal", i.e. < $2.29 \mathrm{IU} / \mathrm{g}$ hemoglobin (G6PD prevalence). Among malaria-infected men $(n=206)$ IC95\% ranged from 3.81 to 4.16 , and among malaria infected women, it ranged from 3.86 to $4.20 \mathrm{IU} / \mathrm{g}$ of hemoglobin. The mean values observed in males (healthy vs. infected) were statistically different $(\mathrm{p}=0.028)$. Only 9.5\% (13/137) of the infected individuals, had G6PD deficiency and this occurred only within the male group. Conclusions: G6PD is relatively high among healthy people (14.8\%) and in the P. vivax infected (9.5\%) male population of Turbo.

Keywords: Glucose-6-phosphate dehydrogenase. Malaria. Plasmodium. Ethnic group. Sex. Primaquine. Colombia.

\section{Introducción}

La glucosa 6-fosfato deshidrogenasa (G6PD) es una enzima hallada en todos los organismos vivientes ${ }^{1}$, donde cataliza el primer paso en la vía de la pentosa fosfato ${ }^{2,3}$. Numerosos estudios han demostrado la importancia de la G6PD en el crecimiento, desarrollo y progreso de la enfermedad celular ${ }^{4}$. Hay más de 400 variantes $^{5}$. La base de la nomenclatura para las variantes se fijó en 1967 por la Organización Mundial de la Salud OMS ${ }^{6}$. La enzima normal se designa B o $\mathrm{B}^{+}$(+ indica actividad enzimática normal); esta es la enzima más común en todos los grupos poblacionales? ${ }^{7}$.

Los eritrocitos maduros carecen de maquinaria celular para obtener energía y sintetizar proteínas y ácidos nucleicos; ellos usan la energía proveniente de la degradación de la glucosa ${ }^{8}$ mediante la glicólisis anaerobia $^{9,10}$. La hemoglobina es muy sensible al daño oxidativo ${ }^{1,11}$ y la deficiencia de G6PD (d-G6PD) aumenta la vulnerabilidad de los eritrocitos a ese estrés ${ }^{10}$.

La d-G6PD es una alteración metabólica (eritroenzimopatía) heredada y ligada al sexo, causada por un defecto en el gen que codifica la enzima $\mathrm{G} \mathrm{PD}^{12}$. Las mujeres con d-G6PD son clínicamente importantes pero el grado de hemólisis es generalmente menor $^{13}$. La d-G6PD se identificó en $1956^{14}$, su determinación cromosómica se conoció en $1958^{15}$ y las variantes electroforéticas se demostraron en $1962^{16}$. Las mujeres son heterocigotas y generalmente son asintomáticas $^{17}$. En la d-G6PD puede ocurrir anemia hemolítica cuya gravedad varía desde leve y asintomática durante toda la vida hasta crisis ocasionales e intensas de anemia ${ }^{8,12}$. La d-G6PD hepática es importante en la hiperbilirrubinemia neonatal.

Las variantes de G6PD están en cinco clases, según la gravedad de la hemólisis ${ }^{7,18}$ (clasificación de la OMS), pero hay otras clasificaciones clínicas ${ }^{11}$. La d-G6PD es la más común de todas las enzimopatías humanas (400 millones de personas ${ }^{19} \mathrm{O}$ $10 \%$ de la población mundial ${ }^{20}$ afectados). La frecuencia masculina de d-G6PD en el 
mundo es muy variable, así (porcentajes): África 0-28, Asia $<1$ a 19, Europa $<1$ a 35, Estados Unidos de América 7-17 en negros; Colombia, Chile, México y Perú: 0 a muy baja; Venezuela 2-12, Islas del Caribe 0 a 13, Brasil 1,7 a 10,18,21,22.

En el mundo hay más de dos mil millones de personas que viven en áreas endémicas de paludismo y en América son 175 millones de personas, en 21 países. En Colombia hay alrededor de 22 millones de sujetos expuestos cotidianamente al riesgo palúdico y en 2001-2002 el país produjo 22\% del total de casos informados en América ${ }^{23,24}$.

Hay coincidencia geográfica entre la presencia de malaria y de defectos eritrocitarios (hemoglobinas anormales, talasemias) y la de d-G6PD ${ }^{25}$. La costa del Pacífico colombiano tiene alta endemia palúdica ${ }^{23} \mathrm{y}$ alta prevalencia de tales defectos ${ }^{26,27}$. En la dG6PD, las mujeres heterocigotas portadoras de esta deficiencia podrían estar protegidas contra la malaria causada por Plasmodium falciparum y las hemoglobinopatías confieren algún grado de protección contra la malaria grave por esta especie ${ }^{26,28,29}$. Desde 1995 han crecido las pruebas sobre las diferencias étnicas en la susceptibilidad a la malaria y de las diferentes adaptaciones a la malaria surgidas en diversas poblaciones $^{30}$. El efecto protector de las variantes polimórficas de las proteínas estructurales específicas eritrocitarias o de las enzimas metabólicas contra los estadios sanguíneos de Plasmodium es una clara ilustración de la modulación genética del humano por el parásito y sugiere coevolución de Plasmodium con su hospedero humano en áreas de endemia palúdica ${ }^{31}$.

Hay varios métodos para diagnosticar la d-G6PD en forma cuantitativa y el método normalizado de Beutler para valoración de la G6PD en hemolizados ${ }^{22,32}$ es el estándar ${ }^{22}$. También hay pruebas cualitativas para la tamización de grandes grupos ${ }^{22,33-37}$, las que requieren examen confirmatorio de un resultado anormal ${ }^{10,38}$. Otro procedimiento de diagnóstico cualitativo rápido usa un nuevo sustrato de formazán (WST-8) y es capaz de detectar mujeres heterocigotas tanto cualitativa como cuantitativamente, según los autores ${ }^{39-41}$. Las pruebas diagnósticas de actividad de G6PD dejan sin detectar alrededor de $50 \%$ de los portadores de deficiencia y las técnicas basadas en reacción en cadena de la polimerasa son más confiables para identificar a los sujetos con deficiencia ${ }^{42}$.

De Colombia, conocemos solo tres publicaciones sobre d-G6PD ${ }^{43-45}$. Las dos primeras son de una misma investigación y refieren una prevalencia entre 1 y $22 \%$ según la etnia y el lugar de residencia; en áreas no palúdicas la frecuencia fue $2 \%$ y en las zonas maláricas fue $9 \%$. El otro trabajo ${ }^{45}$ se hizo en 150 individuos negros del occidente del país e informó $12,7 \%$ de d-G6PD. Los tres informes se refieren a 650 personas y la d-G6PD fue de 13,9\% en negros ( $n=273), 1,4 \%$ en mestizos ( $n=213), 2,5 \%$ en indígenas $(n=119)$, cero en blancos $(n=45)$, lo que implica una prevalencia de $0,1 \%$ en los 650 sujetos. A lo anterior se agrega un informe sobre prevalencia de alelos de G6PD en negros e indígenas noanamas, que refiere que los alelos tuvieron la frecuencia esperada entre los negros, mientras que entre los noanamas la presencia de un individuo con d-G6PD y otros cambios genéticos sugieren un alto nivel de mestizaje ${ }^{46}$.

En América Latina la d-G6PD ha sido poco estudiada, comparada con otros continentes. La consulta (25 diciembre 2006) a Pubmed (palabras clave "G6PD”, "deficiency" y nombre de cada país) y a Lilacs ("glucosa", "fosfato", "deficiencia”) permitió obtener alrededor de 40 y 15 informes, respectivamente, sobre estudios de prevalencia de $\mathrm{d}$ G6PD (Tabla 1) ${ }^{47-66}$. La prevalencia de d-G6PD varió en esos estudios latinoamericanos entre cero y $11,89 \%$, pero en pacientes con anemia hemolítica subió a 30,3\%; la d-G6PD en personas aparentemente sanas fue $2,76 \%$ y en no sanas $3,29 \%$; en negros $11,70 \%$, en mestizos $0,36 \%$, en blancos $4,0 \%$, en amazónicos 5,80\% y en no amazónicos 0,0\%; en neonatos hombres $2,40 \%$ y mujeres $0,85 \%$; en neonatos hombres de México no ictéricos $0,67 \%$ e ictéricos $1,92 \%$. En Brasil hay por lo menos nueve variantes genéticas de G6PD, con la característica de que en los indígenas de ese país casi no existen variantes ${ }^{67}$. 
Tabla 1 - Frecuencia de deficiencia de G6PD en países de América Latina Table 1 - Frequency of G6PD deficiency in Latin America

\begin{tabular}{lcccc}
\hline País & Ref & $\mathrm{n}$ & informes & $\%$ d-G6PD \\
\hline 12 países Latinoamérica y Caribe & 47 & 994 & 1 & 0,8 \\
Brasil & $18,21,48-52$ & 13774 & 8 & 0 a 11,89 \\
Bolivia, Sta Cruz & 53 & 200 & 1 & 0 \\
Perú & 54 & 500 & 1 & 0 \\
Perú, Lima & 55 & 140 & 1 & 0,71 \\
Venezuela, estado Bolívar & 56 & 650 & 1 & 2,9 \\
Cuba, La Habana & $57-60$ & 1572 & 4 & 1,7 a 11,5 \\
México & $61-66$ & 10232 & 6 & 0 a 3,67 \\
\hline & & & & X 3,67 \\
total & & $27923^{\text {b }}$ & 23 & Me 2,00 \\
& & & D.E. 3,59 \\
\hline
\end{tabular}

a prevalencia (porcentaje) de deficiencia de G6PD.

b 27923: total de sujetos sin los 54 de Vaca et al. 1982 que tienen anemia hemolítica. La media, la mediana y la desviación estándar se estimaron sin esas 54 personas. La media no es ponderada por el tamaño del grupo.

c Promedio (X), mediana (Me) y desviación estándar (D.E.) calculados por el autor para los datos de esa columna.

Prevalencia d-G6PD (porcentaje) según datos de las referencias (cálculos nuestros):

Negros $=11,36$; mulatos $=7,58$; mestizos $=0,35$; blancos $=4,53$; amazónicos $=5,80 ;$ no amazónicos $=0,0$.

Neonatos no ictéricos: hombres: 2,59; mujeres: 4,35.

Neonatos ictéricos $=1,42$.

Nosotros estamos ejecutando proyectos de evaluación de la respuesta terapéutica de la malaria vivax al tratamiento con cloroquina y primaquina ${ }^{68,69}$ y parte fundamental de los proyectos es evaluar la actividad de la G6PD antes de iniciar el tratamiento con dosis diaria alta de primaquina.

Estos antecedentes y en especial la escasez de estudios sobre d-G6PD en Colombia dieron lugar a un proyecto de investigación cuyo objetivo fue medir la actividad de la G6PD en dos muestras poblacionales de Turbo de diverso origen étnico: la población masculina, aparentemente sana; pacientes hombres y mujeres con malaria vivax captados en puestos de diagnóstico y sometidos a tratamiento de paludismo. Se requiere conocer más la prevalencia de la d-G6PD, sobre todo ahora que la política colombiana de tratamiento de la malaria vivax dispuso dar más del doble de la dosis diaria de primaquina que se daba antes $(0,6 \mathrm{mg} / \mathrm{kg} /$ día por 7 días, en vez de $0,25 \mathrm{mg} / \mathrm{kg} /$ día por 14 días ${ }^{70}$. La nueva pauta no exige la ejecución previa al tratamiento de una prueba para diagnosticar la d-G6PD ${ }^{70}$, a pesar de que es un criterio mundial hacerlo ${ }^{71-76}$, mucho más si se dan dosis diarias más altas que la estándar. Este informe presenta los resulta- dos de esa investigación en un contexto que revisa la prevalencia de d-G6PD en América Latina, donde en general también son pocos los estudios de prevalencia de la d-G6PD.

\section{Metodología}

\section{Clase estudio, Población de referencia y Tamaño de la muestra}

Como ya se dijo, este informe se refiere a una investigación cuyo objetivo principal fue estudiar la prevalencia de d-G6PD y la actividad de la enzima en dos poblaciones diferentes, representadas por sendas muestras. Para estudiar ambas poblaciones se usó una encuesta de prevalencia, es decir un diseño descriptivo y transversal; los investigadores planificaron y controlaron la clase de observación que en el futuro se haría y la información que captarían. Adicionalmente, se estudió la actividad de G6PD en un grupo de pacientes con malaria vivax que no tenían deficiencia de esta enzima (Tabla 2). En cada sitio visitado para presentar el proyecto, se invitó a las personas o sus representantes a participar y quienes aceptaron se incluyeron en el estudio, hasta completar el tamaño de la muestra. 
Tabla 2 - Prevalencia de deficiencia de G6PD y actividad de la enzima según población estudiada

Table 2 - Prevalence of G6PD deficiency and enzymatic activity according to the population studied

\begin{tabular}{|c|c|c|c|}
\hline \multirow{3}{*}{$\begin{array}{l}\text { Variable } \\
\text { Población }\end{array}$} & \multicolumn{3}{|c|}{ Objetivo del estudio } \\
\hline & \multicolumn{2}{|c|}{$\begin{array}{c}\text { Encuestas de Prevalencia y actividad } \\
\text { enzimática }\end{array}$} & \multirow{2}{*}{$\begin{array}{c}\begin{array}{c}\text { Solo actividad } \\
\text { enzimática }\end{array} \\
\begin{array}{c}\text { Enfermos con malaria } \\
\text { vivax }\end{array}\end{array}$} \\
\hline & Sana & $\begin{array}{l}\text { Enfermos con malaria } \\
\text { vivax }\end{array}$ & \\
\hline Lugar & Turbo, urbana & Turbo, urbana & Turbo, urbana y rural \\
\hline d-G6PD & No conocida & No conocida & No deficiencia \\
\hline Sexo & Hombres & Hombres y mujeres & Hombres y Mujeres \\
\hline Edad (años) (media \pm D.E) & 0,5 a $89(28,1 \pm 17,3)$ & $16-70(31,3 \pm 13,8)$ & $1-70(23,2 \pm 15,7)$ \\
\hline n por grupo & 508 & 137 & 210 \\
\hline n según salud & 508 sanos & \multicolumn{2}{|c|}{347 enfermos } \\
\hline $\mathrm{n}$ total para prevalencia & \multicolumn{2}{|c|}{645} & \multirow[t]{2}{*}{0} \\
\hline $\mathrm{n}$ total & & 855 & \\
\hline
\end{tabular}

- Estudio de prevalencia de d-G6PD en población masculina aparentemente sana

La muestra de 508 hombres se estudió en dos momentos diferentes: en abril de 2005, 256 hombres, y en enero de 2006, otros 252 sujetos. Los participantes se buscaron principalmente en escuelas, colegios, universidad, administración municipal, hospital (empleados). Se incluyeron quienes aceptaron participar. La muestra fue calculada asî ${ }^{77}$ :

$\mathrm{n}=\mathrm{N} Z^{2} \mathrm{p}(1-\mathrm{p}) /\left[\mathrm{Ne}^{2}+\left(\mathrm{Z}^{2} \mathrm{p}(1-\mathrm{p})\right]\right.$,

donde:

$N$ = tamaño de la población de referencia, que era de 61.752 hombres, entre 126.025 habitantes ${ }^{78}$, con $48,99 \%$ como la proporción de hombres allí.

$e=$ error de muestreo, fijado en $5 \%$

$Z$ = intervalo de confianza del $99 \%$, que corresponde a 2,58 unidades $\mathrm{Z}$.

$p=$ proporción esperada de deficiencia de G6PD, fijada en $20 \%$, con base en Restrepo \& Gutiérrez, 1968; Pereira et al., $1986^{44,45}$. $n=61.752 \times 1,96^{2} \times 0,2(1-0,2) /\left[61.752 \times 0,05^{2}\right.$

$\left.+\left(1,96^{2} \times 0,2(1-0,2)\right)\right]$

$\mathrm{n}=423$. Este tamaño se ajustó a 500 sujetos.

La población de referencia para el estudio en la población masculina sana estará constituida por los hombres de cualquier etnia, edad y lugar de residencia en Turbo.
- Estudio de prevalencia de d-G6PD en pacientes con malaria vivax

Los pacientes con malaria vivax se captaron en un puesto de salud de la zona urbana de Turbo, en forma consecutiva según se presentaban al lugar por su propia iniciativa y una vez que aceptaban participar en el estudio. Excepto las gestantes, los pacientes con malaria vivax reciben primaquina en forma rutinaria y según esquemas predefinidos; en esas condiciones, en Colombia no se hace prueba de G6PD como requisito previo a la administración de primaquina ${ }^{79} \mathrm{y}$, en consecuencia, nosotros tampoco lo hicimos. Se trabajó con 137 pacientes adultos (16-70 años). La población de referencia para este estudio estará formada por los adultos, hombres y mujeres de cualquier etnia, edad y lugar de residencia en Turbo, que acuden a los puestos oficiales de malaria.

- Estudio únicamente de actividad de G6PD en niños y adultos con paludismo vivax En otro grupo de enfermos con paludismo vivax se conocía que no eran deficientes en G6PD (según la prueba cuantitativa) y en ellos se midió la actividad de la enzima. Fueron 210 pacientes, de 1-70 años de edad (23,2 $\pm 15,7): 133$ adultos con más de 15 años (32 \pm 15$)$, y 77 menores de 16 años ( $8 \pm 4$ ) (Tabla 2). La población de referencia para estos 
dos últimos grupos es la conformada por hombres y mujeres de cualquier etnia, edad y lugar de residencia en Turbo, que acuden a los puestos oficiales de malaria en la zona urbana del municipio o en el corregimiento El Tres y que tienen malaria vivax.

\section{Medición de la actividad de G6PD}

Al captar la persona se obtuvieron 5 $\mathrm{mL}$ de sangre por punción venosa. Si las muestras no pudieron procesarse en las dos primeras horas, se almacenaron a -20 ${ }^{\circ} \mathrm{C}$ hasta su posterior análisis (3-4 días más tarde). En ambos estudios la actividad de G6PD se midió con la prueba cuantitativa (método normalizado de Beutler para valoración de la G6PD en hemolizados) ${ }^{22,32}$. La medición cuantitativa fue ejecutada por uno de los investigadores y se hizo en dos espectrofotómetros diferentes, calibrados, previamente comprobada su concordancia para la medición. Un aparato fue un Analizer RA 50, modelo 7424 (Bayer) y el otro un Spectronic Genesys 20, modelo 4001/4 (Thermo Electron Corporation). Una actividad menor de 2,29 UI/g Hb se diagnosticó como deficiencia ${ }^{80}$.

\section{Análisis estadístico}

Los datos de actividad de G6PD en cada persona se entraron a una base creada con Epiinfo 6.04; con este programa y con SPSS 14.0 se hizo el análisis de los datos.

Se evaluó la normalidad de cada distribución de frecuencias con la prueba de Kolmogorov-Smirnov (K-S).

Se calcularon las proporciones de prevalencia de deficiencia de G6PD. Para las variables cuantitativas (como actividad de G6PD y edad), se calcularon las medidas estadísticas de resumen (promedio, mediana, desviación estándar, error estándar) y se calculó el intervalo de confianza de 95 para el promedio.

En estos estudios, los grupos comparados son independientes (sexos, etnias, grupos de edad). Se compararon las distribuciones de frecuencias con la prueba chi (ji) cuadrada. Se compararon las medianas de las variables cuantitativas con la prueba no paramétrica de Kruskall y Wallis (K-W). Dos promedios se compararon con la prueba t de Student y más de dos promedios_se compararon con la prueba $\mathrm{F}$ (análisis de varianza anova). Con el procedimiento de Student-Newman-Keuls se compararon pares de promedios después de la comparación global con anova.

Se evaluó la correlación lineal entre variables cuantitativas con el coeficiente de Pearson y la significación estadística del modelo lineal se evaluó con la prueba $\mathrm{F}$.

Todas las decisiones sobre significación estadística se toman con $\mathrm{p}<0,05$.

Se usan estos términos: a: coeficiente de intersección en el modelo lineal $\mathrm{Y}=\mathrm{a} \pm \mathrm{bX}$; anova: análisis de varianza; b: pendiente en el modelo lineal $\mathrm{Y}=\mathrm{a} \pm \mathrm{bX}$; Bartlett $\left(\mathrm{X}^{2}\right)$ : chi cuadrada de Bartlett para homogeneidad de varianzas; D.E: desviación estándar; E.E: error estándar; F: estadístico F de Fisher para el anova; IC95\%(media): intervalo de confianza de 95\% para el promedio; K-W: estadístico de Kruskal y Wallis; LI-IC95\% (media) y LS-IC95\% (media): límites inferior y superior, respectivamente, del IC95\% (media); : valor de probabilidad; $\mathrm{x}+\mathrm{y}$ : promedio \pm desviación estándar; t: estadístico t de Student; \%: porcentaje; \%acum: porcentaje acumulado; Student-Newman-Keuls: prueba para comparar pares de promedios después de la comparación global con anova.

\section{Aspectos éticos}

Cada paciente fue personalmente informado de la investigación, tanto en forma verbal como escrita; se le explicaron los objetivos, beneficios y riesgos; si aceptaba participar debía firmar ante testigos el documento de consentimiento informado, el cual, junto con el proyecto en su conjunto, había sido aprobado por el comité de ética del Centro de Investigaciones Médicas de la Facultad de Medicina de la Universidad de Antioquia. Cuando se trató de menores de 18 años, el anterior procedimiento fue ejecutado con los padres o responsables del menor. 
Os autores, através de documentação assinada e enviada a RBE, declaram não existir nenhum tipo de conflito de interesses.

\section{Resultados}

Los resultados se presentan en tres secciones: A) encuesta de prevalencia en población masculina sana; B) encuesta de prevalencia en población de enfermos de malaria vivax; C) actividad de G6PD en población de enfermos con malaria vivax y sin deficiencia de G6PD.

\section{Encuesta de prevalencia de d-G6PD en hombres sanos}

Se evaluaron 508 personas, con edad entre 0,5 y 89 años $(28,1 \pm 17,3)$. Por etnia, $42 \%$ fueron negros, $55 \%$ mestizos, $3 \%$ blancos y $0,2 \%$ indígenas. La curva de distribución de actividad de G6PD fue gaussiana $($ Kolmogorov-Smirnov $=1,282 ; \mathrm{p}(2$ colas $)=$ 0,075 ) (Figura 1). Hubo valores mínimo de 0,02 y máximo de 12,35 UI/g Hb, promedio 4,33 y desviación estándar 2,06 UI/g Hb; $14,8 \%$ de los sujetos presentaron valores por debajo del "límite normal" (2,29 UI/g Hb), es decir esa es la prevalencia de deficiencia de G6PD en la población (Tabla 3). El IC95\% (media) estuvo entre 4,15 y 4,51 UI/g Hb (con base en el error estándar del promedio: 0,09142). Hubo 41,9\% de hombres con actividad enzimática menor que el límite inferior del IC95\% (media) $(4,15 \mathrm{UI} / \mathrm{g} \mathrm{Hb}) \mathrm{y}$ hubo $47,4 \%$ de las personas con actividad
Población de hombres sanos

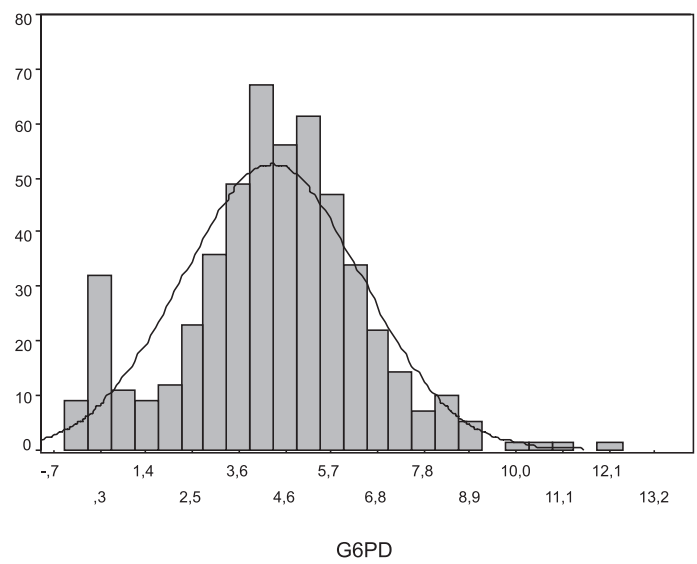

Población de hombres y mujeres con malaria vivax, con G6PD no conocida al ingresar

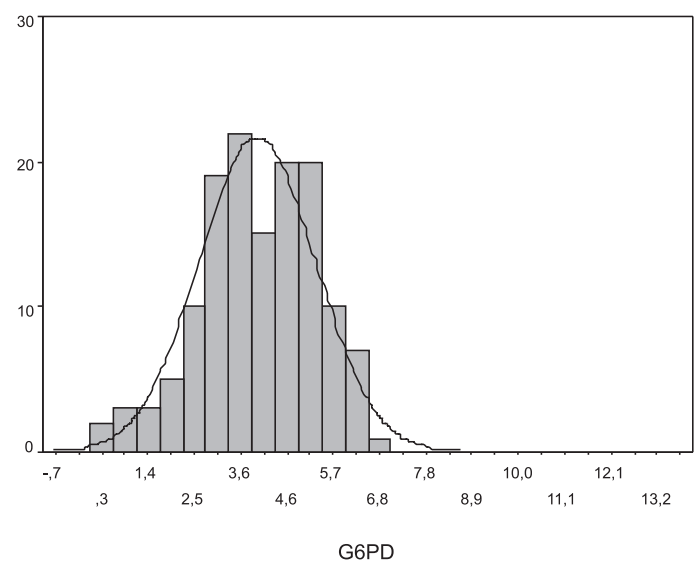

Población de hombres y mujeres con malaria vivax, con G6PD normal al ingresar

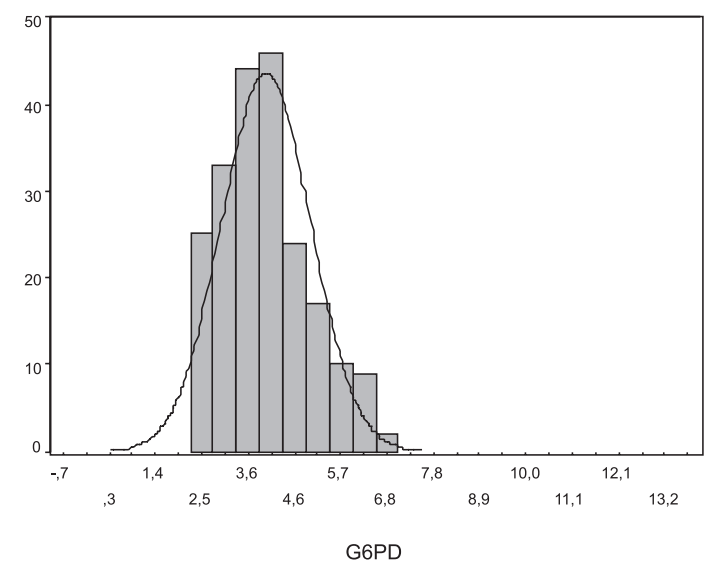

Figura 1 - Histogramas de frecuencias de la actividad de G6PD (UI/g Hb) según tipo de población. Turbo, Colombia, 2005 Figure 1 - Histograms for G6PD activity (UI/g Hb) according to population. Turbo, Colombia, 2005 
Tabla 3 - Actividad de G6PD según población estudiada; Turbo, Colombia, 2005

Table 3 - Activity of G6PD according to population studied; Turbo, Colombia, 2005

\begin{tabular}{|c|c|c|c|c|c|c|c|c|c|}
\hline \multirow[b]{2}{*}{ Estudio } & \multicolumn{6}{|c|}{ Encuestas de prevalencia } & \multirow{2}{*}{\multicolumn{3}{|c|}{$\begin{array}{c}\text { Solo actividad } \\
\text { Hombres y Mujeres niños } \\
\text { y adultos ( } 23,2 \pm 15,7 \text { años) } \\
\text { palúdicos, con G6PD conocida } \\
\text { (malaria vivax population) }\end{array}$}} \\
\hline & \multicolumn{3}{|c|}{$\begin{array}{l}\text { Hombres niños y adultos } \\
(28,1 \pm 17,3 \text { años) sanos } \\
\text { (healthy men) }\end{array}$} & \multicolumn{3}{|c|}{ 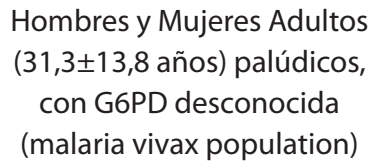 } & & & \\
\hline Prevalencia d-G6PD $\rightarrow$ & \multicolumn{3}{|c|}{$14,8 \%$} & \multicolumn{3}{|c|}{$9,5 \%$} & \multicolumn{3}{|c|}{ No aplica } \\
\hline Actividad (Ul/g Hb) & $\mathrm{n}$ & $\%$ & \%acum & $\mathrm{n}$ & $\%$ & \%acum & $\mathrm{n}$ & $\%$ & \%acum \\
\hline 0,02 a 0,50 & 39 & 7,68 & 7,68 & 2 & 1,46 & 1,46 & & & \\
\hline 0,51 a 1,00 & 9 & 1,77 & 9,45 & 3 & 2,19 & 3,65 & & & \\
\hline 1,01 a 1,50 & 12 & 2,36 & 11,81 & 3 & 2,19 & 5,84 & & & \\
\hline 1,51 a $2,28^{a}$ & 15 & 2,95 & 14,76 & 5 & 3,65 & 9,49 & & & \\
\hline 2,29 a 3,00 & 35 & 6,89 & 21,65 & 25 & 18,25 & 27,74 & 44 & 20,95 & 20,95 \\
\hline 3,01 a 4,00 & 90 & 17,72 & 39,37 & 32 & 23,36 & 51,09 & 63 & 30,00 & 50,95 \\
\hline 4,01 a 5,00 & 114 & 22,44 & 61,81 & 36 & 26,28 & 77,37 & 69 & 32,86 & 83,81 \\
\hline 5,01 a 6,00 & 100 & 19,69 & 81,50 & 28 & 20,44 & 97,81 & 26 & 12,38 & 96,19 \\
\hline 6,01 a 7,00 & 54 & 10,63 & 92,13 & 3 & 2,19 & 100,00 & 8 & 3,81 & 100,00 \\
\hline 7,01 a 8,00 & 20 & 3,94 & 96,07 & & & & & & \\
\hline 8,01 a 9,00 & 15 & 2,95 & 99,02 & & & & & & \\
\hline 9,01 a 2,35 & 5 & 0,98 & 100,00 & & & & & & \\
\hline total & 508 & 100 & & 137 & 100 & & 210 & 100 & \\
\hline
\end{tabular}

a límite inferior de actividad normal: 2,29 Ul/g HB: valores menores a 2,29 indican d-G6PD.

mayor que el límite superior de ese intervalo (4,51 UI/g Hb).

Por etnia (negro, mestizo, blanco) no hubo diferencia significativa en la actividad de G6PD ( $\mathrm{K}-\mathrm{W}=2,567 ; \mathrm{gl}=2 ; \mathrm{p}=0,277)$, pero si por edad ( 9 estratos) $(\mathrm{F}=3,043 ; \mathrm{p}=$ 0,002375): en los menores de 5 años hubo un promedio de 2,85 , que subió a 4,7 - 4,8 entre los 5 y 14 años, luego desciende y llega a 3,4 U.I/g Hb en el grupo 65-89 años (Figura 2-A). Hubo correlación lineal baja y negativa (coeficiente $r=0-0,121$ ) pero significativa entre edad y G6PD ( $\mathrm{F}=7,573$; $\mathrm{gl}=1 ; \mathrm{p}=0,006)$.

\section{Encuesta de prevalencia de d-GPD en pacientes con malaria vivax}

Se examinaron 137 pacientes palúdicos, $64 \%$ hombres, con edad entre 16 y 70 años $(31,3 \pm 13,9)$. Hubo $94 \%$ mestizos, $5 \%$ negros y $1 \%$ blancos. La curva de distribución de G6PD es normal (Kolmogorov-Smirnov = $0,794 ; \mathrm{p}(2$ colas $)=0,553 ; \mathrm{n}=137$ ) (Figura 1$)$. La distribución de frecuencias de la G6PD presentó mínimo de 0,300, máximo de 6,50 y 3,94 $\pm 1,35 \mathrm{UI} / \mathrm{g} \mathrm{Hb}$. El IC95\% (media) es 3,71 a 4,16 UI/g Hb (según el error estándar del promedio: 0,115 UI/g Hb). Hubo $41 \%$ de pacientes con actividad enzimática menor que el límite inferior del IC95\% (media) (3,71 UI/g Hb) y hubo $46 \%$ de las personas con actividad mayor que el límite superior de ese intervalo $(4,16 \mathrm{UI} / \mathrm{g} \mathrm{Hb})$.

Únicamente 9,5\% de los sujetos (13 entre 137 personas), todos hombres, presentaron valores por debajo del "límite normal" de 2,29 $\mathrm{UI} / \mathrm{gHb}$, es decir esa fue la prevalencia de deficiencia de G6PD en los enfermos de paludismo, de cualquier sexo (Tabla 3). El IC95\%(media) estuvo entre 3,84 y 4,08 UI/g Hb (según el error estándar del promedio: 0,062 UI/g Hb). Hubo $47,83 \%$ de pacientes con actividad enzimática menor que el límite inferior del IC95\% (media) (3,84 UI/g Hb) y hubo 48,41\% de las personas con actividad mayor que el límite superior de ese intervalo (4,08 UI/g Hb).

Los valores de G6PD fueron estadísticamente menores $(\mathrm{t}=2,141 ; \mathrm{p}$ ( 2 colas) $=0,034)$ en hombres $(3,78 \pm 1,50)$ que en 
A. Sanos (hombres) [coeficiente r G6PD-Edad: $-0,121 ; p(F)=0,006$ ]

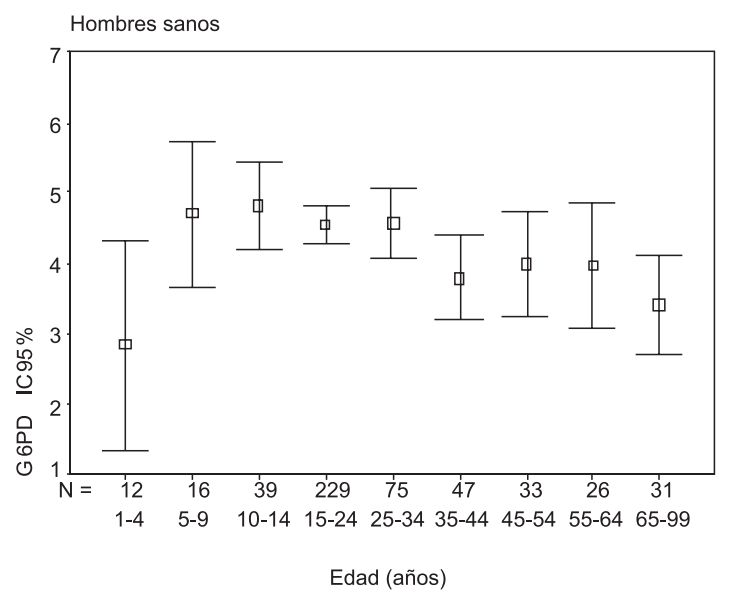

B. Paludismo (hombres y mujeres) con G6PD desconocida

[r G6PD-Edad: $-0,040 ; p(F)=0,641]$

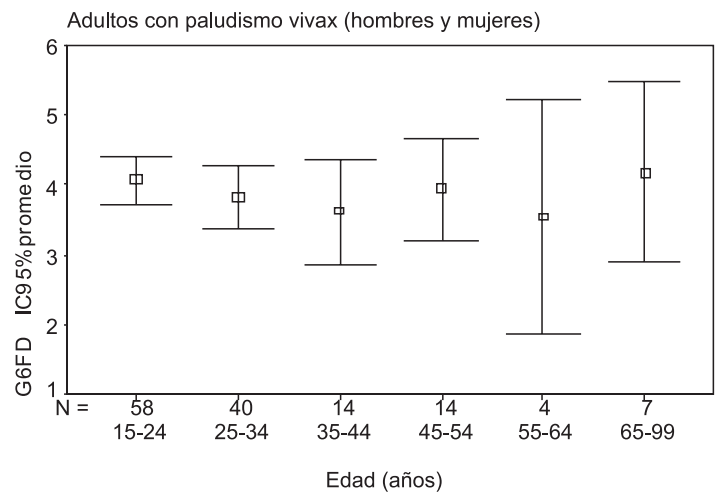

C. Paludismo (hombres y mujeres) con G6PD normal [r G6PD-Edad: $-0,140 ; p(F)=0,043$ ]

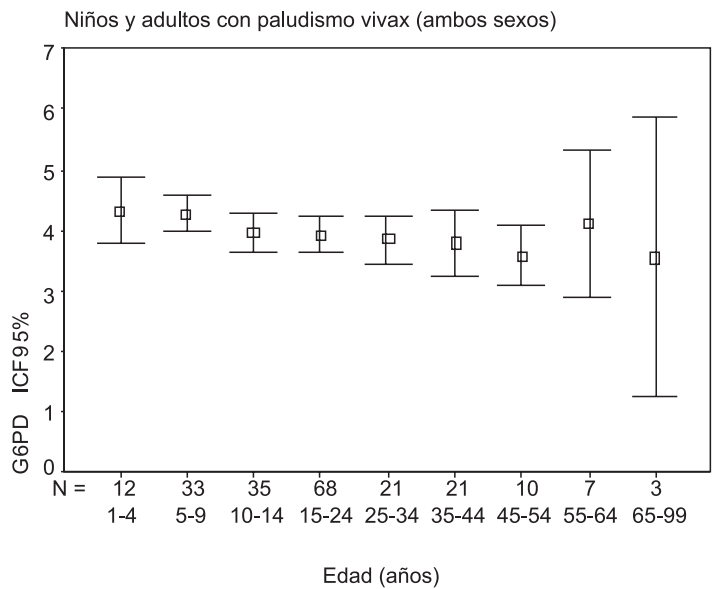

Figura 2 - G6PD (UI/g hemoglobina) por edad y estado de salud (1)

Figure 2 - G6PD (UI/g hemoglobin) by age according to health status.

(1) las barras corresponden al intervalo de confianza $95 \%$ con respecto al promedio, que es el cuadrado central.

mujeres $(4,23 \pm 0,96)$ (Figura 3 -A). No hubo mujeres con menos de 2,29 UI/g Hb (todas mostraron actividad normal). Por etnia (mestizo $v s$. negro) no hubo diferencia significativa en la actividad de G6PD (K - W = $0,017 ; \mathrm{gl}=3 ; \mathrm{p}=0,896524)$, ni tampoco por edad $(\mathrm{K}-\mathrm{W}=2,377 ; \mathrm{gl}=5 ; \mathrm{p}=0,794887)(\mathrm{Fi}-$ gura 2-B). No hubo correlación significativa entre G6PD y edad (anova: $\mathrm{F}=0,230 ; \mathrm{gl}=1$; $\mathrm{p}=0,632)$.

\section{Actividad de GPD en pacientes con malaria vivax y sin deficiencia de G6PD}

Los pacientes estudiados tuvieron como requisito para el ingreso una actividad normal de G6PD (prueba cuantitativa). La curva de distribución de frecuencias de la actividad de G6PD fue normal (Kolmogorov-Smirnov $=1,341 ; p(2$ colas $)=0,055 ; n=210)$. Por sexo, no hubo diferencia en la actividad de G6PD: $4,1 \pm 1,1 \mathrm{UI} / \mathrm{g} \mathrm{Hb}$ hombres y 3,8 $\pm 0,9 \mathrm{UI} / \mathrm{g}$ $\mathrm{Hb}$ mujeres $(\mathrm{K}-\mathrm{W}=2,128 ; \mathrm{p}=0,145)$ (Figura 3-B) pero si por edad (Figura 2-C).

Finalmente se informa que la actividad de G6PD en hombres sanos vs. hombres y mujeres con paludismo mostró que los dos promedios fueron estadísticamente diferentes $(t=3,369 ; p(2$ colas $)=0,001)$, porque hubo mayor actividad de G6PD en la pobla- 

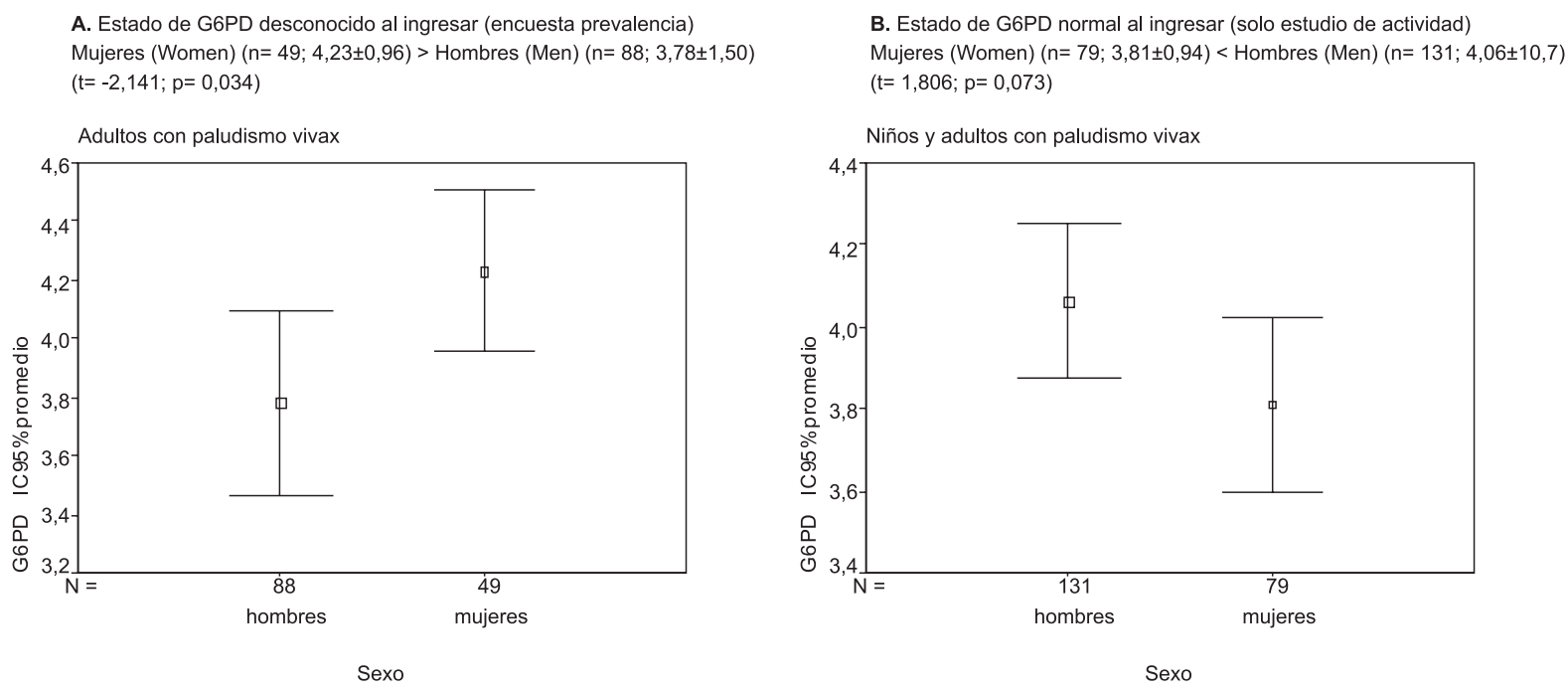

Figura 3 - Actividad de G6PD (UI/g hemoglobina) por sexo en enfermos con malaria vivax ${ }^{(1)}$

Figure 3 - Activity of G6PD (UI/g hemoglobin) in vivax malaria patients, according to gender

(1) las barras corresponden al intervalo de confianza $95 \%$ con respecto al promedio, que es el cuadrado central.

ción masculina sana $(\mathrm{n}=508 ; 4,3 \pm 2,1 \mathrm{UI} / \mathrm{g}$ $\mathrm{Hb})$ que en los enfermos con paludismo ( $\mathrm{n}=347 ; 3,9 \pm 1,2 \mathrm{UI} / \mathrm{g} \mathrm{Hb}$ ). Además, hubo diferencia significativa $(\mathrm{t}=3,040 ; \mathrm{gl}=1 ; \mathrm{p}=$ $0,002)$ entre los promedios de los hombres sanos y los hombres con paludismo: 4,33 \pm 2,06 y $3,95 \pm 1,27$. En el total de sujetos $(\mathrm{n}=$ 855), se apreció un papel dudoso de la edad en la actividad de G6PD, porque la prueba de anova indicó diferencia significativa $(\mathrm{F}$ $=2,545 ; \mathrm{gl}=8 ; \mathrm{p}=0,010$ ), pero la prueba de Student-Newman-Keuls no la halló (p[S-N$\mathrm{K}]=0,216)$. En esas 855 personas, la correlación edad-G6PD fue débil y negativa, pero significativa $(r=-0,106 ; p=0,002)$. En el total de 855 personas, tampoco hubo diferencia significativa entre los promedios de actividad enzimática según la etnia $(\mathrm{F}=0,257$; $\mathrm{gl}=$ $3 ; \mathrm{p}=0,856)$ pero si según el sexo $(\mathrm{t}=2,234$; $\mathrm{p}(2$ colas $)=0,026)$ : hombres 4,21 $\pm 1,86$ ( $\mathrm{n}$ = 727); mujeres $3,97 \pm 0,97(\mathrm{n}=128)$.

\section{Discusión}

La relación estadística de la edad con la actividad de G6PD fue siempre débil y negativa y, al final, confusa: en sujetos sanos (hombres) el modelo lineal es significativo $(p=0,006)$, así como en personas enfermas (hombres y mujeres) y con G6PD normal $(p=0,043)$, pero en enfermos de uno y otro sexo sin la condición de normalidad prevista en la G6PD, el modelo no fue significativo $(\mathrm{p}=0,641)$ (Figura 2). Llamó la atención que, en los hombres sanos, los grupos extremos (0-4 y 65-89 años) presentaron los valores más bajos (3,60 UI/g hemoglobina), mientras que en los otros estuvieron, en general, alrededor de 4,10 , pero esto no es así en las otras poblaciones.

La actividad de G6PD según el sexo mostró diferencia significativa entre los pacientes con paludismo cuyo estado de G6PD se desconocía al ingresar al estudio (encuesta de prevalencia), pero no en aquellos cuyo estado se sabía que era normal cuando entraron. Este hallazgo es lógico, en el sentido de que el último grupo supone actividad normal y, en cambio, en el primero si hay deficientes probablemente será entre los hombres, como en efecto sucedió. Este hallazgo concuerda con la información general ${ }^{1-6,37}$. En Colombia, se ha informado una prevalencia de d-G6PD, en sujetos aparentemente sanos, entre 1 y $22 \%$ según la etnia y el lugar de residencia; en áreas no palúdicas fue $2 \%$ y en las zonas maláricas fue 9\%; en negros varió de 13 a $22 \%$, en blancos $2,5 \%$ y en indígenas catíos $0 \%{ }^{43,44}$. En negros sanos de Cali se encontró 
$12,7 \%$ de d-G6PD ${ }^{45}$. En el corregimiento Bajo Calima, en Buenaventura, se calculó, en 1984, una prevalencia de d-G6PD de $13,7 \%{ }^{27}$. En el presente informe, nosotros hallamos una prevalencia de d-G6PD de $14,8 \%$ en hombres sanos, $3,75 \%$ hombres palúdicos y $0 \%$ en mujeres palúdicas, todos ellos en Turbo, zona endémica de malaria. Esa diferencia significativa en la prevalencia de d-G6PD entre hombres sanos y hombres con paludismo concuerda con la idea general de que la d-G6PD eritrocitaria protege del paludismo ${ }^{25,27,30,31}$; además, nosotros encontramos que los afectados por malaria tienen menos actividad enzimática que quienes no poseen paludismo.

La información obtenida podrá usarse para definir ajustes en las políticas de tratamiento de la malaria, especialmente en la malaria por P. vivax, donde el protocolo más usado en el mundo indica que debe darse primaquina (PQ) por 14 días para prevenir las recurrencias palúdicas; este medicamento es un potente oxidante $\mathrm{y}$ al ser ingerido por personas con d-G6PD induce crisis hemolíticas de gravedad variable, que inclusive pueden ser fatales. La PQ debe usarse solo en personas con actividad normal de G6PD, sin embarazo y el medicamento debe tomarse con alimentos ${ }^{71-76}$. A su capacidad curativa, la PQ une su elevada eficacia para prevenir la malaria cuando se usa como quimioprofiláctico primario, según lo hemos demostrado recientemente mediante un metanálisis ${ }^{81}$. Los efectos adversos de la PQ son pocos, leves y pasajeros, cuando se usa en las personas indicadas. Un estudio en adultos con dosis de $0,6 \mathrm{mg} / \mathrm{kg} /$ día por 28 días (es decir, $30 \mathrm{mg} /$ día y dosis total de $420 \mathrm{mg}$ ) solo halló efectos adversos en los 13 pacientes con deficiencia de G6PD y la dosis alta debió suspenderse en únicamente $31 \%$ de ellos por una significativa reducción del hematocrito ${ }^{82}$.

En el panorama de la malaria vivax en Colombia y en América Latina, urge conocer la frecuencia de d-G6PD en las diferentes etnias de los países y crear programas estables de detección precoz (tamización) de la deficiencia desde la edad de recién nacidos.

\section{Referencias}

1. Fonseca D, Mateus H, Silva C, Contreras N, Restrepo C. Deficiencia de glucosa 6-fosfato deshidrogenasa. Aspectos generales de la eritroenzimopatía más frecuente en el mundo. Acta Med Colomb 2005; 30: 59-64.

2. Mehta A, Mason PJ, Vulliamy TJ. Glucose-6-phosphate dehydrogenase deficiency. Bailliere's Clinical

Haematology 2000; 13: 21-38.

3. Nomenclature Committee of the International Union of Biochemistry and Molecular Biology (NC-IUBMB). Enzyme Nomenclature. EC 1 Oxidoreductases EC 1.1 to EC 1.3. [sitio en internet]. IUBMB Enzyme Nomenclature. EC 1.1.1.49. Disponible en: http://www. chem.qmul.ac.uk/iubmb/enzyme/EC1/1/1/49.html. [1 febrero 2007].

4. Ho HY, Cheng ML, Chiu DT. G6PD--an old bottle with new wine. Chang Gung Med J 2005; 28: 606-12.

5. OMIM Online Mendelian Inheritance in Man; Johns Hopkins University. [sitio en internet]. Glucose-6Phosphate Dehydrogenase; G6PD. Disponible en: http://www.ncbi.nlm.nih.gov/entrez/dispomim. cgi?id=305900. [1 febrero 2007].
6. World Health Organization. Nomenclature of glucose-6phosphate dehydrogenase in man. Bull WHO 1967; 36: 319-22.

7. Organización Mundial de la Salud OMS. Tratamiento de las hemoglobinopatías y los trastornos asociados. Geneve: Ginebra: OMS; 1972. Serie Informes Técnicos 509.

8. Acosta-Sánchez T, Núñez DP, Suárez-Luengo M. Anemia hemolítica por deficiencia de G6PD y estrés oxidativo. Rev Cubana Invest Biomed 2003; 22: 186-91.

9. Stiene EA. Red cell enzyme deficiencies: A review. Am J Med Tech 1972; 38: 454-61.

10. Frank JE. Diagnosis and management of G6PD deficiency. Am Fam Physician 2005; 72: 1277-82

11. Luzzatto L. Glucose 6-phosphate dehydrogenase deficiency: from genotype to phenotype. Haematologica 2006; 91:1303-6.

12. Lica L. Glucose-6-Phosphate Dehydrogenase Deficiency. [sitio en internet] Caremark. Disponible en: http:// healthresources.caremark.com/topic/printview. [12 octubre 2006]. 
13. Chan TK. Glucose-6-Phosphate Dehydrogenase (G6PD) Deficiency: A review. [sitio en internet] http://www. cchi.com.hk/specialtopic/case1/case1.htm. [12 octubre 2006].

14. Carson PE, Flanagan CL, Ickes CE, Alving AS. Enzymatic deficiency in primaquine-sensitive erythrocytes. Science 1956; 124(3220): 484-5.

15. Childs B, Zinkham W, Browne EA, Kimbro EL, Torbert JV. A genetic study of a defect in glutathione metabolism of the erythrocytes. Bull Johns Hopkins Hosp 1958; 102: 21-37.

16. Boyer SH, Porter IH, Weilbacher RG. Electrophoretic heterogeneity of glucose-6-phosphate dehydrogenase and its relationship to enzyme deficiency in man. Proc Nat Acad Sci USA 1962; 48: 1868-76.

17. Beutler E, Yeh M, Fairbanks VF. The normal human female as a mosaic of X-chromosome activity. Studies using the gene for G6PD deficiency as a marker. Proc Natl Acad Sci USA 1962; 48: 9-16.

18. Compri MB, Saad STO, Ramalho AS. Investigação genético-epidemiológica e molecular da deficiência de G-6-PD em uma comunidade brasileira. Cad Saúde Pública 2000; 16: 335-42.

19. rialto. An introduction to G6PD deficiency [sitio en internet]. Disponible en: http://www.rialto.com/g6pd/. [1 diciembre 2005].

20. medicinenet.com. Glucose-6-phosphate dehydrogenase deficiency. MedicineNet.com. Diagnosis [sitio de internet]. Disponible en: http://www.answers.com/ topic/glucose-6-phosphate-dehydrogenase-deficiency. [21 junio 2006].

21. Katsuragawa TH, Soares-Gil LH, Stábile RG, Pires MG, Bonini-Domingos CR. Avaliação da incidência da deficiência de Glicose-6-Fosfato Desidrogenase (G6PD) e perfil hematológico em indivíduos de uma região de Rondônia. Rev bras hematol hemoter 2004; 26: 268-73.

22. Organización Mundial de la Salud OMS. Normalización de las técnicas de estudio de la glucosa 6 -fosfato deshidrogenasa. Ginebra: OMS; 1967. Serie Informes Técnicos 366 .

23. Carmona-Fonseca J: La malaria en Colombia, Antioquia y las zonas de Urabá y Bajo Cauca: panorama para interpretar la falla terapéutica antimalárica. Parte 1. Iatreia 2003; 16: 299-318. Disponible en: http:// medicina.udea.edu.co/Investigacion/Grupos/malaria/ index.htm

24. Carmona-Fonseca J: La malaria en Colombia, Antioquia y las zonas de Urabá y Bajo Cauca: panorama para interpretar la falla terapéutica antimalárica. Parte 2. Iatreia 2004; 17: 34-53. Disponible en: http://medicina. udea.edu.co/Investigacion/Grupos/malaria/index.htm

25. Cavalli-Sforza Ll, Bondmer WF. The Genetics of Human Populations. New York: Dover Publications; 1999.
26. Colombia, Ministerio de Salud, Instituto Nacional de Salud. Hemoglobinopatías en Colombia. Informe Quincenal Epidemiológivo Nacional (IQEN) 1997; 2: 171.

27. Méndez F, Moyano M. Defectos eritrocíticos y densidad de la parasitemia en pacientes con malaria por Plasmodium falciparum en Buenaventura, Colombia. Rev Panam Sal Pub 2005; 18: 25-32.

28. Hill A. Malaria resistance genes: a natural selection. Trans Roy Soc Trop Med Hyg 1992; 86: 225-6, 232.

29. Cappadoro M, Garibaldi G, Obrien E, Turrini F, Mannu F, Ulliers D et al. Early phagocytosis of glucose6-phosphate dehydrogenase (G6PD) deficient erythrocytes parasitized by Plasmodium falciparum may explain malaria protection in G6PD deficiency. Blood 1998; 92: 2527-34.

30. Kwiatkowski DP. How malaria has affected the human genome and what human genetics can teach us about malaria. Am J Hum Genet 2005; 77: 171-92.

31. Min-Oo G, Gros P. Erythrocyte variants and the nature of their malaria protective effect. Cell Microbiol 2005; 7: 753-63.

32. Beutler E. Glucose-6-phosphate dehydrogenase deficiency. Diagnosis, clinical and genetic implications. Am J Clin Pathol 1967; 47: 303-11.

33. Beutler E. A series of new screening procedures for pyruvate kinase deficiency, glocuse-6-phospate dehydrogenase deficiency and glutathiones reductase deficiency. Blood 1966; 28: 553-62.

34. Beutler E, Mitchell M. Special modifications of the fluorescent screening method for glucose-6-phosphate dehydrogenase deficiency. Blood 1968; 32: 816-8.

35. Blume KG, Beutler E. Detection of glucose-phosphate isomerase deficiency by a screening procedure. Blood 1972; 39: 685-7.

36. Beutler E, Blume KG, Kaplan C, Lohr GW, Valentine WN. International Committee for Standarization in Haematology: recomemended screening test for glucose-6-phosphate dehydrogenase (G-6-P-D) deficiency. Brit J Haematol 1979; 43: 465-7.

37. Beutler E. G6PD deficiency. Blood 1994; 84: 3613-36.

38. Gregg XT, Prchal JT. Red cell enzymopathies. In: Hoffman R (ed). Hematology: basic principles and practice. $4^{\text {th }}$ ed. Philadelphia: Churchill Livingstone; 2000. p. 657-60.

39. Tantular IS, Iwai K, Basuhi S, Horie T, Htay HH, Matsuoka H et al. Field trials of a rapid test for G6PD deficiency in combination with a rapid diagnosis of malaria. Trop Med Internat Health 1999; 4(4): 245-50.

40. Tantular IS, Kawamoto F. An improved, simple screening method for detection of glucose-6-phosphate dehydrogenase deficiency. Trop Med Int Health 2003; 8: 569-74. 
41. Jalloh A, Tantular IS, Pusarawati S, Kawilarang AP, Kerong H, Lin K et al. Rapid epidemiologic assessment of glucose-6-phosphate dehydrogenase deficiency in malaria-endemic areas in Southeast Asia using a novel diagnostic kit. Trop Med Int Health 2004; 9: 615-23.

42. Pronk-Admiraal CJ, van Zwieten R, Roos D. Moleculargenetic studies of carrier stage of glucose-6-phosphate dehydrogenase deficiency. Ned Tijdschr Geneeskd 1994; 138: 1715-9. Resumen obtenido en Pubmed.

43. Arias S, Restrepo A. Deficiencia de G6PD en Colombia: valores enzimáticos normales y patológicos en Colombia. Tribuna Médica 1966; 262: 9-12.

44. Restrepo A, Gutiérrez E. The frequency of glucose-6phosphate dehydrogenase deficiency in Colombia. Am J Hum Genet 1968; 20: 82-5.

45. Pereira FD, Rosero C, Aristizabal T. Deficiencia de glucosa 6 fosfato deshidrogena (G-6PD). Colomb méd 1986; 17: 16-8.

46. Monsalve MV, Espinel A, Groot de Restrepo H, Calvo M, Suarez MC, Rodríguez A. Frequency of five genetic polymorphisms in two populations of Colombia. Rev bras genét 1987; 10: 247-51.

47. Acosta T, Suárez M, Núñez V, Marín LC, Cordero A. Efecto hemolítico de la cloroquina en estudiantes deficientes de glucosa-6-fosfato deshidrogenasa. Rev Cubana Invest Biomed 2003-b: 22: 180-5.

48. Azevedo WC, Silva ML, Grassi MC, Azevedo ES. Glucose6-phosphate dehydrogenase deficiency in a general hospital of Salvador, Bahia, Brazil. Rev Bras Pesqui Med Biol 1978; 11: 49-52.

49. Barraviera B, Alves Meira D, Marques Pereira PC, Marcondes J, Mendes RP, Curi PR, Grecchi Pirolla J. A. Malária no municipio de Humaitá, estado do Amazonas. XX. Alterações eletrocardiográficas em doentes, antes e após o tratamento. Arquivos brasileiros de medicina. Arq bras med 1987; 61: 235-9.

50. Garlipp CR, Ramalho AS. Aspectos clínicos e laboratoriais da deficiência de desidrogenase de 6-fosfato de glicose (G-6-PD) em recém nascidos brasileiros. Rev bras genét 1988; 11: 717-28.

51. Neto EC, Portal L, Ferreira LF. G6PD deficiency in an unselected Brazilian population. Southeast Asian J Trop Med Public Health 1999; 30(S2): 87.

52. Castro S, Weber R, Dadalt V, Tavares V, Giugliani R. Prevalence of G6PD deficiency in newborns in the south of Brazil. J Med Screen 2006; 13: 85-6.

53. Cardozo L, Zuna H, Urjel R, Saavedra F. Estudio de las causas de anemia en embarazadas de la Maternidad Percy Boland de la ciudad de Santa Cruz, 1985. Bol cient CENETROP 1985; 11: 58-67.

54. Reyes P, Páucar J, Muñoz E, Ramos A. Estudio de la deficiencia de Glucosa 6 Fosfato Deshidrogenasa (G-6P-D) en un grupo de mestizos. Rev serv sanid fuerzas polic 1985; 46: 8-12.
55. Ruiz W, Ulloa V, Bailón O. Prevalencia de la deficiencia de glucosa-6-fosfato deshidrogenasa en donadores voluntarios de sangre que acuden a los hospitales nacionales Cayetano Heredia y Arzobispo Loayza. LimaPerú. Rev méd hered 1997; 8: 11-8.

56. Godoy G, Valero J, Rivas R. Deficiencia de glucosa 6 fosfato deshidrogenasa en maláricos del Estado Bolívar. Gac méd Caracas 1997; 105: 30-3.

57. Estrada M, González R. Ictericia neonatal y deficiencia de glucosa-6-fosfato deshidrogenasa en la Ciudad de la Habana. Rev Invest Clin 1983; 35: 297-9.

58. González-González OL, Hidalgo-Calcines PC. Alteraciones metabólicas eritrocitarias en recién nacidos a término con ictericia fisiológica: glutatión reducido y glucosa 6-fosfato deshidrogenasa. Medicentro 1986; 2: 89-93.

59. Hidalgo PC, González OL, Rodríguez M, Castellanos T. Análisis del polimorfismo genético de la G-6FD eritrocitaria en la región central de Cuba. Medicentro 1987; 3: 149-57.

60. Martínez-Pérez JL, Hadad-Meléndez P. Síndrome hemolítico por primaquina y deficiencia de glucosa 6 fosfato deshidrogenasa. Rev cuba med trop 1989; 41: 299-306.

61. Vaca G, Hernandez A, Ibarra B, Velazquez A, Olivarez N, Sanchez-Corona J, Medina C, Cantu J M. Deteccion de errores congenitos del metabolismo en 1.117 pacientes estudiados por sospecha de enfermedad hereditaria. Arch invest méd 1981; 12: 341-8.

62. Vaca G, Ibarra B, Hernandez A, Velazquez AL, GonzalezQuiroga G, Romero F et al. Screening for inborn errors of the erythrocyte metabolism in Northwestern Mexico. Acta Anthropogenet 1982; 6: 255-64.

63. Velázquez AL, Rico NG, Ibarra B, Blancarte R, Cardosa J, Fonseca S et al. Eritroenzimopatías hereditarias en neonatos con hiperbilirrubinemia. Bol méd Hosp Infant Méx 1985; 42: 466-9.

64. González-Quiroga G, Walle-Cardona M, Ortiz-Jalomo R, Garza-Chapa R. Deficiencia de deshidrogenasa de la glucosa -6- fosfato (G6PD) en varones neonatos en Monterrey, Nuevo León. Rev méd IMSS 1985; 23: 247-50.

65. González-Quiroga G, Ramírez del Río JL, Ortiz-Jalomo R, Garcia-Contreras RF, Cerda-Flores RM, Mata-Cardenas BD, Garza-Chapa R. Frecuencia relativa de deficiencia de glucosa-6-fosfato deshidrogenasa en recién nacidos ictéricos en el área metropolitana de Monterrey, Nuevo Leon. Arch Invest Med (Mex) 1990; 21: 223-7.

66. González-Quiroga G, Ramírez-Del Río JL, Cerda-Flores RM, Garza-Chapa R. Frequency and origin of G-6-PD deficiency among icteric newborns in the metropolitan area of Monterrey, Nuevo Leon, Mexico. Gene Geogr 1994; 8:157-64.

67. Weimer TA, Salzano FM, Westwood B, Beutler E. Molecular characterization of glucose-6-phosphate dehydrogenase variants from Brazil. Hum Biol 1993; 65: 41-7. 
68. Carmona-Fonseca J, Álvarez G, Blair S. Malaria por Plasmodium vivax: curación del ataque agudo con tres dosis diferentes de primaquina y dosis fija de cloroquina; Antioquia (Colombia), 2003-2004. Biomédica 2006; 26:353-65.

69. Álvarez G, Piñeros JG, Tobón A, Ríos A, Maestre A, Blair S, Carmona-Fonseca J. Efficacy of three chloroquineprimaquine regimens for treatment of Plasmodium vivax malaria in Colombia. Am J Trop Med Hyg 2006; 75: 605-9.

70. República de Colombia, Ministerio de la Protección Social. Plan de implementación de combinaciones terapéuticas con derivados de artemisinina en áreas de alta transmisión de malaria en Colombia. Bogotá: Minprotección; junio 2006. Documento preliminar.

71. Clayman CB, Arnold J, Hockwald RS, Yount EH, Edgcomb JH, Alving AS. Toxicity of primaquine in Caucasian. JAMA 1952; 149: 1563-8.

72. Baird JK, Rieckmann KH. Can primaquine therapy for vivax malaria be improved? Trends Parasitol 2003-a; 19: 115-20.

73. Baird JK, Fryauff DJ, Hoffmann SL. Primaquine for prevention of malaria in travelers. Clin Infect Dis 2003-b; 37: 1659-67.

74. López-Antuñano FJ. Is primaquine useful and safe as true exo-erythrocytic meronticidal, hyponociticial and gametocidal antimalarial drugs? Salud Pub Mex 1999; 41: 410-9.
75. Kain KC, Shanks GD, Keystone JS. Malaria chemoprophylaxis in the age of drug resistance. I. Currently recommended drug regimens. Clin Infect Dis 2001; 33: 226-34.

76. Carmona-Fonseca J. Profilaxis primaria con primaquina para el paludismo: revisión. Anuario Enfermedades Infecciosas (Medellín) 2004-b; 2: 51-84.

77. Martínez-Bencardino C. Muestreo. Bogotá: Ecoe, 1984. p. 45-7.

78. Colombia, DANE Departamento Administrativo Nacional de Estadística. Colombia. Proyecciones de población, por área, según municipios [sitio en internet]. Disponible en: www.dane.gov.co. [1 diciembre 2006].

79. Colombia, Ministerio de Salud. Guía de atención clínica para el diagnóstico y tratamiento de la malaria. Bogotá: Minsalud; 1999.

80. Menéndez-Capote R, Díaz-Pérez L, Luzardo-Suárez C. Hemólisis y tratamiento con primaquina. Informe Preliminar. Rev cuba med trop 1997; 49: 136-8.

81. Carmona-Fonseca J. La primaquina tiene alta eficacia en la quimioprofilaxis primaria simple antipalúdica. Metanálisis. Iatreia 2006; 19: 244-59.

82. Silachamroon U, Krudsood S, Treeprasertsuk S, Wilairatana P, Chalearmrult K, Mint HY et al. Clinical trial of oral artesunate with or without high-dose primaquine for the treatment of vivax malaria in Thailand. Am J Trop Med Hyg 2003; 69: 14-8.

Recebido em: 18/05/07 Versão final reapresentada em:05/12/07 Aprovado em: 27/02/08 